\title{
Adding flavours to our MOFs
}

Alessia Bacchi ${ }^{1}$, Davide Balestri ${ }^{1}$, Stefano Canossa ${ }^{1}$, Nicola Demitri ${ }^{2}$, Paolo Pio Mazzeo ${ }^{1}$, Paolo Pelagatti ${ }^{1}$, Patrick Scilabra ${ }^{3}$

${ }^{1}$ Dipartimento SCVSA, Universita' Di Parma, Parma, Italy, ${ }^{2}$ Elettra-Sincrotrone Trieste S.C.p.A., Trieste, Italy, ${ }^{3}$ Dipartimento Giulio Natta, Politecnico di Milano, Milano, Italy

E-mail: alessia.bacchi@unipr.it

MOFs are highly versatile materials that are made by connecting metal ions with prefixed coordination geometry with rigid ligands acting as spacers, hence affording three-dimensional coordination polymers. The accurate design of the building units allows to design porous MOFs, obtaining cavities of considerable size, which usually accommodate loosely bound solvent molecules. The scope of this work is to find a systematic way to embed small molecular aggregates inside porous crystalline materials, with the multiple aims to explore the structural aspects of nanoconfinement, and of the stabilization of guest molecules inside the cavities of the structure. The feasibility of this approach stems from both the recently developed crystalline-sponge method that describes the structural determination of single molecules trapped inside a microporous framework [1]. The guest that we are here considering for inclusion in MOFs pores are some important compounds for the human health and nutrition which occur as liquids at room temperature, such as eugenol (a major component of clove oil), carvacrol (extracted from the oregano essential oil), thymol (present in the oil of thyme); these are part of a naturally occurring class of compounds known as biocides, with strong antimicrobial attributes. In order to embed these guest in crystalline materials we needed to carefully tailor the MOF cavities; thus we designed a small library of new ligands which could afford flexible MOF architectures. We were able to characterize a series of new materials which are able to release the guests in a controlled way, opening the way to engineer additives for active food packaging.

In particular the structure and inclusion properties of PUM168 will be discussed. This new heteroleptic MOF shows triple interpenetration, and nevertheless can accommodate all the guests belonging to the oil family by exhibiting a remarkable flexibility; the selectivity towards mixtures will also be discussed. In the Figure the triple interpenetration of the MOF architecture is shown on the left, and an example of MOF...guest interaction with carvacrol via hydrogen bonds is shown on the right.

[1] Inokuma Y., et al., (2013), Nature, 495, 461-466

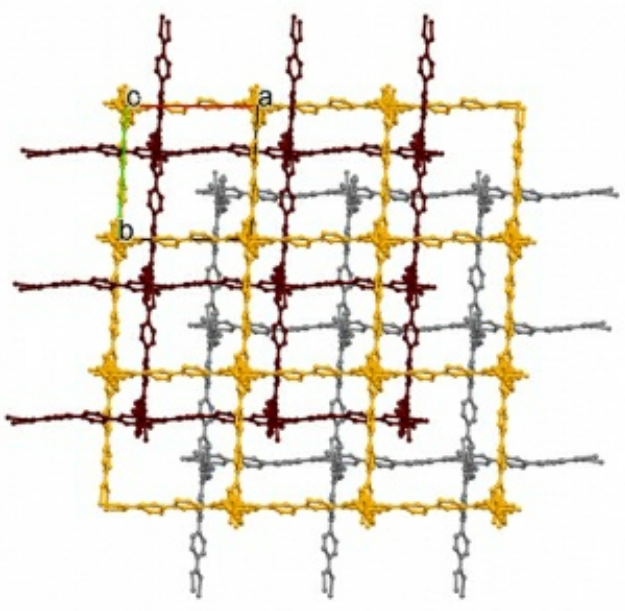

PUM168

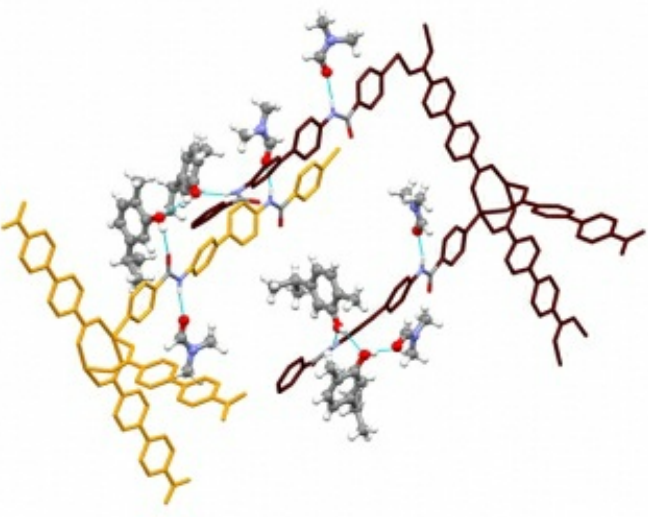

carvacrol@PUM168

Keywords: $\mathrm{MOF}$, guest inclusion, nutraceuticals 